\title{
Developing a Framework for Adopting Artificial Intelligence
}

\author{
Sanjay Mohapatra and Ajit Kumar
}

\begin{abstract}
Artificial Intelligence has the ability to predict outcomes accurately and with reliability. The techniques have been used in several industries and domains. However, documenting results from different research that were conducted have not been documented. Also, most of the research have been carried out in developed countries and not much work have been published from other economies. As a result, there is a need to develop proper research background so that application of AIs can be sustainable and effective. The purpose of this study is to critically review different studies that have adopted AI in several domains, so that a theoretical framework guide for researchers and practitioners can be developed. This framework will also establish future trends in the said research area. From online databases, relevant articles and extracts were retrieved and were systematically analyzed. Using these inputs, a framework was developed. The findings of this study show that there is a gap between research work done and documentation available. The present applications of AI techniques require model based approach that brings in consistency in research as well as for industry. A paradigm shift in the framework based approach could lead to achieving a sustainable practice.
\end{abstract}

Index Terms-Artificial intelligence, framework, theoretical study, AI applications.

\section{INTRODUCTION}

One of the challenges of Artificial Intelligence is defining human and robot interaction models (HRI) [1]. AI seems to be at every domain and hence modelling of human and robot interaction (HRI) requires integration of humans and human cognition. It also requires acquiring, representing, manipulating abstract knowledge at the human level. This integration will help to make decisions which will result into physical and tangible actions.

Over decades, AI systems have been improving. Research on applications of AI systems have been going on [2]-[8]. Particularly AI applications in assembly and manufacturing sector have been developing [9]. These will improve in flexibility, configurability and reliability of the systems. Machine intelligence combines a wide variety of advanced technologies to give machines an ability to learn, adapt, make decisions and display new behaviours [10]. This is achieved using neural networks and expert systems [11]. There are also improvements in areas of self-organizing neural networks [12], fuzzy logic [13] and genetic algorithms [14]. Also machine learning has been developed through its application to many areas such as factory level assembly [15], building statistical models for predictions

Manuscript received December 5, 2018; revised February 20, 2019.

Sanjay Mohapatra and Ajit Kumar are with Xavier Institute of Management, Bhubaneswar, India (e-mail: sanjay_mohapatra@yahoo.com, ajitmaskara@gmail.com).
[16], computer processing speed [17]

Artificial Intelligence based machines are consistent and better human performances in new manufacturing methods such as rapid-manufacture and the manufacture of composites [18]. However, the problem lies that we do not have hardware capabilities to integrate AI with MicroElectro-Mechanical-System (MEMS) devices. This is primarily because of limitations in capabilities to manufacture MEMS with AI capabilities. If they can be effectively introduced into MEMS devices then we should be able to combine our brain power with computer capacity to create a powerful AI [19].

\section{LITERATURE REVIEW}

The recent advances in AI come are related to "machine learning," which involves programming computers to learn from data or past decisions [20]. For example, in a basket of groceries, machine learning can be used to identify apples based on their color and shape. However, there are other objects that are apple-like in both color and shape [21]. In order to avoid mistakes in identifying apples, programming need to be refined so that the machine can identify apples in finer detail. This will, however, increase complexity in programming. In these complex environments, machine learning is most useful. In one type of training, the machine is shown a set of pictures with names attached. It is then shown millions of pictures that each contains named objects, only some of which are apples. Using statistical models, the machine notices correlations and learns on its own that apples are often red. Using correlates such as color, shape, texture, and, most important, context, the machine rebuilds information from past images of apples to predict and identify objects.

AI requires investment in technology and hence there needs to be a clear managerial decision on the same. The value of prediction will be realized through faster, accurate predictions with higher productivity [22]. The value of prediction becomes more valuable when data is more widely available and more accessible. The computer revolution has enabled huge increases in both the amount and variety of data. As data availability expands, prediction becomes increasingly possible in a wider variety of tasks.

While predicting, the machine usually anticipates what will happen in the future [23]. For example, machine learning can be used to predict whether a bank customer will default on a loan. Similarly, in healthcare, using symptoms, machines can develop medical diagnosis and predict the presence of a disease. With advances in computational speed, data storage, data collection, data retrieval processes have become faster and reliable. The sensors are used for easier 
and faster data collection. The algorithms have combined with these data to reduce the cost of machine learning based predictions. Using multi- layer approach, image recognition and language translation have become faster and accuracy has increased.

Artificial intelligence (AI) modeling techniques that use artificial neural network (ANN) techniques are designed to replicate human brains [24]. The ANN technique has been used for prediction, pattern recognition, classification, process control, nonlinear mapping and data analysis [25]. The ANN technique has been successfully applied in business transactions and has produced more accurate and reliable predictions [26].

In Finance domain, AI techniques will help in appraising different customers and their applications for loans and advances [27]. At the same time, there is a need to identify the past contributions, research gap(s) and also to predict the future direction.

There are literatures available related to application of AIs to finance [28], business [29], HIV epidemic [30], market segmentation [31], reliability analysis of steel construction, civil engineering [32], [33], clothing comfort [34] and construction management [35], amongst others. However, using these techniques in banking sector can lead to better productivity, consistency and customer satisfaction. Research needs to be conducted to come up with a framework that can be used for continuous investment in areas of customer relationship management, providing resolutions to issues raised by customers and complying with regulatory requirements [36].

There has not been similar effort to document in the finance and banking domain. To this end, this study aims to review literature related $\mathrm{AI}$ related studies in order to present the framework for the application of AI in banking sector and also to establish the trend in this research area. This would provide both AI researchers and finance professionals with a guide and insight into the application of the AI techniques.

Artificial Intelligence based machines are consistent and have improved performances [37]. However, the problem lies that we do not have hardware capabilities to integrate AI with Micro-Electro-Mechanical-System (MEMS) devices. This is primarily because of limitations in capabilities to manufacture MEMS with AI capabilities.

\section{GAPS IN LITERATURE}

Literature available in AI dates back to 1930s. Work is still going in full swing in different aspects of AI; however, there are areas where more work need to be done. One such area is developing industry specific frameworks for employing AI in business transactions. This framework, when developed, will provide rational behind employing AI effectively. This will also make the implementation of AI a sustainable one. There are not enough case studies in Banking sectors particularly which hinders understanding where AI can be used and where it cannot be.

Therefore, through this research, we will develop a framework for banking industry and then validate this through a case study.

\section{RESEARCH OBJECTIVE}

From the discussions, it is felt that there needs to be a good theoretical base on which AI can be applied in the industry. This will make it robust and sustainable. The ability to predict accurately with high degree of reliability has led to its application in banking sectors. However, a large percentage of such previous studies have focused on other domains in developed economies, and at the same time, effort has not been put into documenting its research trend in banking domain. The purpose of this paper is to critically review the studies that adopted AI for prediction and develop a framework and present an application guide for researchers and practitioners through a case study, and also establish the trend in this research area.

\section{Methodology}

To develop framework, a systematic review of the available literature will be an efficient and appropriate approach. By going through literature, we can understand the subject better and present a systematic output. This output in form of a paper can be used to develop a reliable knowledge base which can be useful to both researchers and industry practitioners. Hence, this study has adopted a literature review approach to develop the framework. For this, we selected the papers that had applied Artificial Intelligence in different industries. These papers were downloaded from online databases and search engines such as ProQuest (ABI Complete), Ebsco, Emerald, and Google Scholar. Articles that were published in journals, conference proceedings and sections of books were retrieved. From these papers, studies that did not have application of AI were excluded during the search process. While making search, publication date was left open so as to retrieve the earliest paper that adopted AI in different domains. The keywords used for the search were "artificial intelligence," "AI," "framework," "artificial intelligence appraisal" and "industry using artificial neural network."

At the end, 31 relevant papers were retrieved from the search. Of the 31 articles, 28 are journal articles, three are conference proceedings, while only one is a book. This search approach helped to identify trends in research related to AI studies in different domains.

Thorough readings of the selected articles were made to select articles that actually adopted the AI technique in different domains. From the filtered articles, meaningful extracts were done for writing this paper. The extracted information included the names of authors, the authors' affiliations, the sample size of data used for the analysis, input variables, the year of publication, the journals in which the articles were published, the country of origin of the articles, AI technique adopted for the development of the models. This information was eventually analyzed in order to draw meaningful conclusions from the review of the literature.

\section{PROPOSED FRAMEWORK}

As discussed in Methodology section, literatures were reviewed in a scientific manner to derive the variables and 
arrive at the framework. The following paragraphs discuss different variables that were derived for arriving at the framework.

The recent advances in AI come are related to "machine learning," which involves programming machines and computers to learn from data insights. For example, in a basket of groceries, machine learning can be used to identify fruits based on their sizes, colors and shapes. However, there are other objects that could be similar in both color and shape. In order to avoid mistakes in identifying fruits, programming need to be refined so that the machine can identify apples in finer detail. This will, however, increase complexity in programming. In these complex environments, solution can be achieved through machine learning.

While predicting, the machine usually predicts the future. For example, it can be used to predict whether a customer who has taken a loan from bank will default on a loan. Similarly, in healthcare, using symptoms, machines can develop medical diagnosis and predict the presence of a disease. With advances in computational speed, data storage, data collection, data retrieval processes have become faster and reliable. The sensors are used for easier and faster data collection. The algorithms have combined with these data to reduce the cost of investment in machine learning based technology. Using multi- layer approach, image recognition and language translation have become faster and accuracy has increased.

Artificial Neural network techniques replicate thinking power of humans. These techniques will be part of AI modeling techniques and were used for multi-layer recognition, classification of information, recognition of pattern, controlling processes, data analysis and nonlinear mapping. These techniques have proven to be successful in business transactions and have been able to predict results accurately, consistently and with reliability. In Finance domain, AI techniques will help in appraising different customers and their applications for loans and advances.

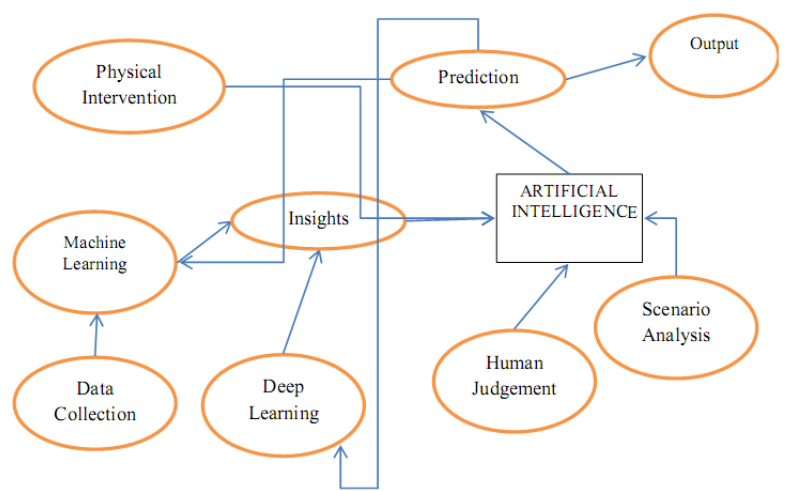

Fig. 1. Developing a framework for adopting artificial intelligence.

Using these techniques in banking sector can lead to better productivity, consistency and customer satisfaction. Research needs to be conducted to come up with a framework that can be used for continuous investment in areas of customer relationship management, providing resolutions to issues raised by customers and complying with regulatory requirements. There has not been similar effort to document in the finance and banking domain. Based on these variables and techniques, the proposed framework for AI has been illustrated in Fig. 1.

\section{THE MANAGERIAL CHALLENGE}

There will be continuous research on Artificial Intelligence, which will increase the level of accuracy in prediction. As artificial intelligence technology improves, AIs will replace human beings in predictive roles. At that point of humans roles will be for providing judgement which AIs will not be able to predict. These judgements are typically decisions that relate to exceptional use cases which have for which algorithms are not designed. Some examples of these judgements are related to ethics, creative and visual artistic taste, emotional and spiritual intelligence, breaking down work to simple processes and tasks. As a result, there will be a need for people who can take new and responsible decisions (requiring ethical judgment), defining touch points for customers and engaging employees in different decision making processes. These will require creativity, emotional intelligence and judgement and will lead to identifying new business opportunities. Hence, there will be integration of $\mathrm{AI}$ and humans with humans taking higher roles of judgements. In this scenario, prediction by AIs will become an input to automating different tasks. The entire output will be function of prediction by AIs, raw data, insights and judgements of humans. Future automation will require automatic data collection through sensors such as Internet of Things (IOTs), Chatbots and prediction by machine learning (both components of $\mathrm{AI}$ ).

The role of the manager will involve deciding how to make utilize AIs so that he gets best of prediction capabilities from AIs and also gets judgements from human beings. For this to happen, the manager should know what need to be predicted by using data with levels of accuracy. For this, AI agents would provide prediction for mundane and repetitive kind of jobs. The higher the accuracy in prediction will mean higher investment in technology related to AI. But whenever the goal is not clear and there is ambiguity, then managers' judgment will become a particularly valuable complement to prediction technology.

\section{FUTURE RESEARCH}

At present, the most common predictions used in business are related to inventory management, repetitive customer care and demand forecasting. However, AI has been used successfully to recognize image, auto pilot driving, and language translations. The main challenges for organizations will be upgrading skills of employees to make judgment related decisions. Another challenge will be to assess the processes where AI technologies can be adopted so that processes can be re-engineered.

\section{REFERENCES}

[1] S. Sarkar, S. Mohapatra, and J. Sundarakrishnan, "Assessing impact of technology based digital equalizer programme on improving student learning outcomes," Education and Information Technologies, 2017.

[2] D. A. Sanders, "Viewpoint - Force sensing," Industrial Robot: An International Journal, vol. 34, no. 4, p. 268, 2007. 
[3] D. A. Sanders, "Controlling the direction of "walkie' type forklifts and pallet jacks on sloping ground," Assembly Automation, vol. 28, no. 4, pp. 317-324, 2008.

[4] D. A. Sanders, "Environmental sensors and networks of sensors," Sensor Review, vol. 28, no. 4, pp. 273-274, 2008.

[5] D. A. Sanders, "Progress in machine intelligence," Industrial Robot: An International Journal, vol. 35, no. 6, pp. 485-487, 2008

[6] D. A. Sanders, "Ambient intelligence and energy efficiency in rapid prototyping and manufacturing," Assembly Automation, vol. 29, no. 3 , 2009.

[7] D. A. Sanders, "A pointer device for TFT display screens that determines position by detecting colours on the display using a colour sensor and an artificial neural network," Displays, vol. 30, no. 2, pp. 84-96, 2009.

[8] D. A. Sanders, "Recognizing shipbuilding parts using artificial neural networks and Fourier descriptors," in Proc. IMechE, Part B: Journal of Engineering Manufacture, 2009, vol. 223, no. B3, pp. 337-342.

[9] G. Riva, F. Vatalaro, F. Davide, and M. Alcañiz, Ambient Intelligence in Practice: Future Perspectives and Applications, Amsterdam: IOS Press, 2005, pp. 237-264

[10] S. Mohapatra, "Improvised process for quality through quantitative project management: An experience from software development projects," International Journal of Information and Communication Technology, 2010

[11] S. Mohapatra, "Better healthcare at reduced cost through electronic integration of patient care data," International Journal of Electronic Healthcare, 2009.

[12] S. Mohapatra, "Requirement management - controlling quality at the upstream in commercial software project management," International Journal of Applied Engineering Research, 2015.

[13] S. Mohapatra, "Business school education and technology-a case study," Education and Information Technologies, 2015.

[14] U. Chakraborty. (2018). We know very little about human brain Artificial neural network vs. human centre of cognition. [Online] Available: https://medium.com/@utpal_bob/we-know-very-littleabout-human-brain-artificial-neural-network-vs-66e4ac20a508

[15] R. B. Abidoye and A. P. C. Chan, "A survey of property valuation approaches in Nigeria," Property Management, vol. 34, no. 5, pp. 364-382, 2016

[16] Emerald Group Publishing Limited, "An intelligent inspection solution for smarter seating," Assembly Automation, vol. 28, no. 2, p. 173, 2008.

[17] W. Wang, M. Song, Z. Y. Zhang, and M. Richardson, "Synthesis and characterization of high nickel-containing mesoporous silica via a modified direct synthesis method," Journal of Non-Crystalline Solids, vol. 352, no. 21-22, pp. 2180-2186, 2006.

[18] Z. Y. Zhang and M. O. W. Richardson, "Low velocity impact induced damage evaluation and its effect on the residual flexural properties of pultruded GRP composites," Composite Structures, vol. 81, no. 2, pp. 195-201, 2007.

[19] D. J. Harrison and D. A. Sanders, "Imagineering - promoting creativity in engineering education," Engineering Education, vol. 2, pp. 45-49, 1992.

[20] J. W. Hinks, H. Cawte, D. A. Sanders, A. Hudson, and C. Dockree, "Prediction of flow rates and stability in large scale airlift reactors," Water Science and Technology, vol. 34, no. 5-6, pp. 51-57, 1996.

[21] J. W. Hinks, H. Cawte, D. A. Sanders, O. Fenske, G. A. Poland, and G. E. Tewkesbury, "Model for the prediction of liquid volumetric flow rates in large scale airlift reactors," in Proc. 3rd International Conference on Water and Waste Treatment, BHR Group Conference Series, 1995, Cranefield, no. 17, pp. 125-133

[22] M. A. Boden, The Creative Mind: Myths and Mechanisms, New York: Basic Books, 1990

[23] K. Binsted, "Machine humour: An implemented model of puns," Ph.D. Thesis, University of Edinburgh, 1996.

[24] S. Mohapatra, R. Padhi, and S. Mohanty, Study of motivationa factors post-implementing ERP (SAP) solutions in National Aluminium Company Limited extending the technology acceptance model at organisation level (TAMO)," International Journal of Management and Enterprise Development, 2015.
[25] S. Mohapatra, "Using integrated information system for patien benefits: A case study in India," International Journal of Healthcare Management, 2015.

[26] A. Gegov, "Application of computational intelligence methods for intelligent modelling of buildings: applications and science in soft computing," in A. Loffi and J. Garobaldi, Eds. Advances in Soft Computing, Springer, Berlin, pp. 263-270, 2004.

[27] M. J. Goodwin, D. A. Sanders, G. A. Poland, and I. J. Stott, "Navigational assistance for disabled wheelchair-users," Journal of Systems Architecture, vol. 43, no. 1-5, pp. 73-79, 1997

[28] J. Gribbin, Richard Feynman: A Life in Science, New York, NY: Dutton, 1997, p. 170

[29] F. Fuerst, X. Liu, and C. Lizieri, A Commercial Real Estate Index for an Emerging Market: The Case of Beijing, Cambridge: University of Cambridge, 2016, pp. 1-20.

[30] S. M. Guru, S. Fernando, S. Halgamuge, and K. Chan, "Intelligent fastening with A-BOLT technology and sensor networks," Assembly Automation, vol. 24, no. 4, pp. 386-393, 2004.

[31] A. D. Hudson, D. A. Sanders, H. Golding, G. E. Tewkesbury, and H Cawte, "Aspects of an expert design system for the wastewater treatment industry," Journal of Systems Architecture, vol. 43, no. 1-5, pp. 59-65, 1997.

[32] A. D. Hudson, D. A. Sanders, G. E. Tewkesbury, H. Cawte, and J Hinks, "Simulation of a high recirculation airlift reactor for steadystate operation," Water Science and Technology, vol. 34, no. 5-6, pp 59-66, 1996.

[33] M. Kress, "Intelligent internet knowledge networks: processing of concepts and wisdom," Information Processing \& Management, vol 44, no. 2, pp. 983-984, 2008

[34] T. W. Manikas, K. Ashenayi, and R. L. Wainwright, "Genetic algorithms for autonomous robot navigation," IEEE Instrumentation \& Measurement, vol. 10, no. 6, pp. 26-31, 2007.

[35] C. Matuszek, D. Fox, and K. Koscher, "Following directions using statistical machine translation," in Proc. the 5th ACM/IEEE International Conference on Human-Robot Interaction, 2010, pp. 251-258.

[36] M. M. Mooya, "The education and professional practice of valuers in South Africa: A critical review," Property Management, vol. 33, no. 3, pp. 245-274, 2015

[37] S. Mohapatra and S. Murarka, "Improving patient care in hospital in India by monitoring influential parameters," International Journal of Healthcare Management, 2016.

Sanjay Mohapatra has done his B.E. from NIT Rourkela, MBA from XIMB, M.Tech from IIT Madras, India and PhD from Utkal University. Dr. Mohapatra has more than 27 years of combined industry and academic experience. He has consulted many organizations in different domains such as Utilities, Banking, Insurance and healthcare sectors. His teaching interests are in IT Strategy and Management Information Systems and research interests are in the area of IT enabled processes. He has authored or co-authored twenty six books, more than sixty two papers in national and international referred, indexed journals besides publications in different conferences. His contact details and list of publications can be found at http://ximb.academia.edu/sanjaymohapatra/.

Ajit Kumar completed his postdoctoral in human computer interaction from National Central University, Taiwan, and $\mathrm{PhD}$ in health informatics from Taipei Medical University, Taiwan. He has been involved in teaching, research, and software industries more than 13 years across four countries -

Taiwan, Libya, India, and Nigeria. He has worked with sophisticated healthcare information and communication technology that includes legacy mainframe to ubiquitous cloud computing. He is interested in learning, teaching, and researching in areas, such as human-computer interaction, computer applications in health care, usability of healthcare software, and software engineering. He has published seventeen papers in peer-reviewed journals, and presented twenty conference papers in various national and international conferences. His contact details and list of publications can be found at http://ajit-kumar.weebly.com/. 